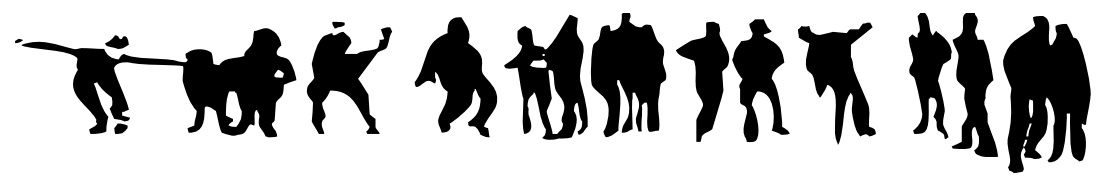

\section{Un regard rétrospectif sur le développement professionnel des enseignants dans le modèle de l'École éloignée en réseau}

Christine HAMEL, Thérèse LAFERRIÈRE (Université Laval,
Québec) Sandrine TURCOTTE (Université du Québec en Outaouais, Outaouais) Stéphane ALLAIRE (Université du Québec à Chicoutimi, Chicoutimi), chercheurs réguliers au Centre de recherche et $d$ 'intervention sur la réussite scolaire (CRIRES)

- RÉSUMÉ • Le développement professionnel (DP) des enseignants est un des facteurs importants pour la réussite scolaire des élèves. Plusieurs dispositifs de DP existent afin de soutenir les enseignants, mais peu d'entre eux donnent des résultats reconnus dans leur pratique en classe de même que chez les élèves. Au Québec, l'initiative de l'École éloignée en réseau, qui vise à enrichir l'environnement d'apprentissage des petites écoles rurales à l'aide des technologies collaboratives, a permis d'offrir plusieurs occasions de DP aux enseignants participants avec le support de TIC, entre autres, la visioconférence et un forum électronique. Le présent article porte, à l'aide du cadre théorique de Guskey (Guskey, 2003), un regard rétrospectif sur le DP des enseignants qui mettent en ouvre cette innovation afin de nommer leurs réactions au dispositif, les apprentissages et l'utilisation qu'ils en ont faits, le soutien organisationnel nécessaire, et finalement les résultats observés chez les élèves.

MOTS-CLÉS • Développement professionnel, technologies de télécollaboration, dispositif de formation, innovation, apprentissage

ABSTRACT • Teacher professional development (PD) is an important factor of student academic success. Several PD devices exist to support teachers, but few of them provide visible results in their classroom practice as well as on student learning. In Quebec, the initiative of the Remote Networked Schools, which aims to enrich the learning environments of small rural schools by using collaborative technologies, has provided several PD opportunities for participating teachers, with the support of information and communication technologies (ICT) such as video conferencing and an electronic forum. This article, using the theoretical framework of Guskey (Guskey, 2003), provides a retrospective on the professional development of the teachers implementing this innovation, in order 
C. HAMEL, T. LAFFERIÈRE, S. TURCOTTE , S.ALLAIRE

to identify their reactions to the device, their understanding of the device and its different uses, the organizational support required, and ultimately,students outcomes.

- KEYWORDS - Professional development, collaborative technologies, sociotechnical designs, innovation, learning with technology 


\section{Introduction}

\subsection{Le développement professionnel des enseignants par des activités professionnalisantes}

Le développement professionnel ou l'apprentissage des enseignants est maintes fois nommé comme l'un des critères importants pour l'amélioration de la réussite scolaire des jeunes (Borko, 2004) ; (VillegasReimers, 2003). Le concept de développement professionnel est polysémique, car il est soit le fait d'activités professionnalisantes proposées soit de processus individuels. Nous avons choisi d'adopter la perspective professionnalisante du développement professionnel chez les enseignants puisqu'elle est porteuse de sens pour notre étude de par sa posture constructiviste et sa description de l'action de l'enseignant détenteur d'un savoir en construction (Uwamariya et Mukamurera, 2005). Compte tenu de ce choix, plusieurs dispositifs de développement professionnel existent afin, d'une part, de permettre aux enseignants d'améliorer leur pratique et d'acquérir de nouveaux savoirs et aussi, d'autre part, pour répondre aux demandes ministérielles, par exemple lors de l'implantation d'un nouveau curriculum (Guskey, 2002) ; (Lieberman et PointerMace, 2008). Toutefois, ces dispositifs de développement professionnel ${ }^{1}$ offerts aux enseignants sont souvent rendus caducs par les difficultés que rencontrent les enseignants à réinvestir les contenus dans leur classe ou encore parce qu'ils ne répondent pas aux principaux besoins et intérêts des enseignants (Hunzicker, 2012) ; (Quick et al., 2009) ; (Tate, 2009). Ainsi, des recherches ont démontré le peu d'efficacité des formules de développement professionnel de type séminaire alors que les enseignants assistent à des présentations un jour durant (Flores, 2005) ; (Fullan, 1995) ; (Liebermann et Pointer Mace, 2008) ; (Wayne et al., 2008) et ont peu de possibilités d'échanger avec leurs collègues afin de faire des liens avec leur pratique en classe. De plus, les contenus ont souvent peu d'ancrage dans des résultats de recherche ou encore ne sollicitent pas la participation d'enseignants à une recherche qui pourrait en découler (Liebermann et Pointer Mace, 2008). En outre, la pertinence des communautés d'enseignants pour l'apprentissage et le développement professionnel a beau être démontrée, elles demeurent l'exception dans le système scolaire (Collinson et al., 2009).

C'est dire que le dispositif de développement professionnel le plus fréquemment utilisé peut être contradictoire à son contenu par exemple, lorsqu'on demande aux enseignants de se centrer sur les besoins et les 
capacités de leurs élèves pour les faire apprendre tout en n'appliquant pas cette idée à leur propre développement professionnel (Hawley et Valli, 2007). C'est dire aussi que le développement professionnel des enseignants aurait intérêt à être davantage centré sur le partage de pratiques exemplaires ou les apprentissages actifs et caractérisé par une bonne cohérence processus/contenu afin d'en augmenter les effets sur les apprentissages des élèves (Garet et al., 2001)Un autre aspect assez souvent mis en évidence est l'absence de suivi après les activités de développement professionnel offertes, ce qui contribuerait à ce que peu d'éléments de contenu soient réinvestis dans la pratique des enseignants. Le suivi sous forme, entre autres, d'accompagnement pédagogique serait à considérer comme partie intégrante des dispositifs de développement professionnel mis en place pour les enseignants afin d'assurer un plus grand réinvestissement des meilleures pratiques en classe (Goderva-Shaikh, 2010) ; (Ingvarson, 2005) ; (Penuel et al., 2007). Ainsi, pour que des activités de développement professionnel parviennent à transformer les pratiques des enseignants, il faudrait miser sur une bonne dose de soutien et d'accompagnement, sans négliger l'exercice d'une certaine pression pour faire entreprendre cette transformation (Guskey, 2002)

À cet égard, les technologies de télécollaboration sont des outils puissants afin de soutenir les communautés d'enseignants et assurer un accompagnement et un suivi du lieu même de leur pratique (Hamel, 2003) ; (Marx et al., 1998). Néanmoins, le manque de temps et le manque de ressources financières sont souvent les arguments évoqués pour offrir des activités de développement professionnel aux enseignants sous leur formule habituelle, soit des journées de formation au cours desquelles un contenu spécifique est abordé avec les enseignants - ici caractérisée de formule «juste-au-cas ». En contraste, le suivi et l'accompagnement des enseignants dans une formule de type « juste-à-temps » supposent plutôt que l'enseignant reçoive du soutien au moment où il en a besoin dans sa pratique en classe. Des outils technologiques tels que la visioconférence sont maintenant disponibles pour permettre une telle formule. Par ailleurs, cette formule est très peu présente dans le système scolaire étant donné que la première est plus facile à mettre en place et à encadrer et parait moins dispendieuse. Qu'en est-il des dispositifs de développement professionnel mis en place sur de larges territoires?

\subsection{Un contexte favorable à un dispositif innovant en développement professionnel des enseignants}


Au Québec, l'immensité du territoire apporte son lot de défis afin d'assurer une égalité de chances et de succès en éducation. En effet, environ $30 \%$ des écoles sont de petite taille (moins de 100 élèves) et elles contribuent, entre autres, à préserver la vitalité des villages. Ce sont des écoles qui ont des classes multiâges (de deux à quatre niveaux par classe) et peu de ressources professionnelles disponibles. En outre, elles connaissent un fort mouvement de personnel et sont souvent situées en milieu défavorisé. On peut alors se questionner sur la qualité de l'environnement éducatif de ces petites écoles lorsque peu de ressources sont disponibles pour l'enseignement et le soutien à l'apprentissage. C'est à partir de cet état de situation qu'en 2001, le ministère de l'Éducation a mis en place l'initiative de l'École éloignée en réseau (ÉÉR) afin d'explorer comment l'utilisation des technologies de l'information et de la communication (TIC) pouvait permettre d'imaginer autrement l'avenir de la petite école rurale québécoise. Un organisme spécialisé dans le transfert de connaissances et dans l'innovation, le Centre francophone d'informatisation des organisations (CEFRIO) a coordonné l'initiative de l'ÉÉR jouant un rôle précieux auprès des chercheurs, du Ministère de l'Éducation de même que des commissions scolaires ${ }^{2}$ participantes.

Les TIC choisies furent un système de visioconférence (iVisit et VIA) et un forum électronique de coélaboration de connaissances (Knowledge Forum $[\mathrm{KF}]$ ) rendues disponibles directement dans les classes participantes. Il s'agissait d'utiliser ces nouveaux outils afin d'augmenter les possibilités d'interactions humaines pour faire apprendre les élèves des petites écoles. Pour des raisons pédagogique et politique, l'avenue de l'enseignement à distance fut écartée d'emblée. Ainsi à tous les jours, encore aujourd'hui, des élèves et enseignants de classes distantes collaborent en planifiant et en réalisant des activités d'apprentissage authentiques ancrées dans le programme de formation de l'école québécoise à l'aide de ces outils. À titre d'exemple, des élèves de classes distantes peuvent réaliser des résolutions de problèmes en mathématiques à l'aide de la visioconférence ou encore écrire une histoire collective. Dans le forum électronique, les élèves écrivent en collaboration afin de comprendre un problème complexe; cela se produit surtout en ce qui concerne les sciences (pourquoi les feuilles changent de couleur à l'automne?) ou l'univers social (qu'est-ce qu'une société non démocratique?). Si, lors des premières années de mise en œuvre de l'ÉÉR, les activités réalisées servaient surtout des apprentissages périphériques (par exemple, la fête de l'Halloween), ce n'est plus le cas depuis plusieurs années maintenant, et ce pour une majorité de classes 
actives. Ce qui s'effectue à l'aide des outils contribue donc de très près au développement des connaissances et des compétences prescrites par le Programme de formation de l'école québécoise. Les enseignants et les élèves les plus avancés mettent en place dans leur classe une pédagogie qui vise la coélaboration ou la création de connaissances (Scardamalia et Bereiter, 1991). Pour parvenir à de tels résultats, le design de recherche et d'intervention mis en place auprès des participants a joué un rôle important (Hamel et al., 2012a) ; (Laferrière et al., 2004a) ; (Turcotte et al., 2009). Entre autres, une équipe de recherche-intervention (ÉRI) est disponible dans une salle de visioconférence durant les heures de classe afin de répondre à des questions et à des problèmes des enseignants directement du lieu de leur classe, au moment même où ils surviennent, et ce depuis 2002.

Depuis le début, les participants ont été invités à contribuer, de manière créative, à la mise en œuvre de l'École éloignée en réseau, soit à s'engager dans une démarche de design collaboratif de l'innovation. Ainsi, à partir des activités réalisées dans les classes, le modèle de l'École éloignée en réseau s'est défini et raffiné. Au fil des années en est ressorti un modèle qui renforce la capacité de préserver la qualité de l'environnement d'apprentissage de la petite école de village. Ainsi, lorsque des conditions favorables sont réunies (Turcotte et Hamel, 2008), les enseignants sont capables de mettre en place des activités d'apprentissage centrées sur les élèves en utilisant les TIC. À cet égard, les environnements d'apprentissage des élèves s'en sont trouvés améliorés tant au niveau de la quantité que de la qualité des interactions de même que des apprentissages spécifiques dans des domaines comme le français langue d'enseignement et les sciences et technologie.

Si l'ensemble du système scolaire est impliqué pour réussir la mise en œuvre de l'École éloignée en réseau, le cœur de cette innovation, c'est la classe. Ce sont donc les enseignants qui ont été les professionnels les plus sollicités puisque c'est dans leur classe que se déroulaient les activités et les projets d'apprentissage en réseau. À l'aube de l'utilisation de la visioconférence en classe, ils ont planifié des activités d'apprentissage en collaboration. Un contexte favorable existait dans tout le Québec du fait de la réforme du curriculum pour lequel nous passions d'une approche centrée sur des objectifs à une approche centrée sur le développement de compétences (MEQ, 2006). 
Lors de la mise en œuvre imposante de cette innovation sociale qu'est l'École éloignée en réseau pour les petites écoles, il a fallu promouvoir et soutenir le développement professionnel des enseignants. Le présent article vise à cerner le développement professionnel offert aux enseignants durant les quatre phases de l'ÉÉR à partir des cinq niveaux de développement professionnel définis par Guskey (Guskey, 2003) dans ses travaux axés sur l'innovation en milieu scolaire : les réactions des enseignants, les apprentissages des enseignants, le soutien organisationnel, l'utilisation des savoirs nouvellement acquis par les enseignants et les résultats des élèves. Cet article se veut un regard rétrospectif et une synthèse en ce qui concerne le développement professionnel chez les enseignants qui ont participé à l'initiative ÉÉR entre 2002 et 2010 puisqu'il nous permet de prendre du recul à partir d'un ensemble de résultats disponibles.

\section{Cadre théorique}

Nous avons retenu le modèle de Guskey pour présenter les principaux résultats obtenus pendant huit ans en ce qui concerne le développement professionnel des enseignants participants de l'ÉÉR. Ce modèle comporte cinq niveaux critiques pour apprécier les impacts des dispositifs de développement professionnel offerts aux enseignants. Dans le contexte de l'École éloignée en réseau, les activités de développement professionnel $\mathrm{du}$ dispositif visaient à utiliser les TIC pour enrichir l'environnement d'apprentissage de la petite classe multiâge en milieu rural. Ce dispositif de développement professionnel s'est transformé tout en se complexifiant au fur et à mesure que les enseignants développaient leur capacité dans l'usage des TIC pour faire apprendre les élèves. Nous présentons ci-après les cinq niveaux de Guskey et les mettons en lien avec les caractéristiques du dispositif de développement professionnel disponible dans l'ÉÉR.

\subsection{Niveau 1 : Les réactions des enseignants}

À ce niveau, il s'agit de saisir les réactions des enseignants envers les activités de développement professionnel qui leur ont été offertes. C'est à ce niveau qu'il est le plus facile d'obtenir de l'information à propos de ce que pensent les enseignants à propos de ce qu'ils ont appris. Est-ce qu'ils ont trouvé cela utile? Est-ce pertinent pour leur pratique? Est-ce que les formateurs étaient compétents? Ce premier niveau est souvent le seul niveau qui soit évalué ou étudié et il s'exprime dans nombre d'études sous le vocable du niveau de satisfaction des participants. Dans notre contexte, les enseignants participaient à certaines activités formelles et non formelles en lien avec l'ÉÉR. Nous recueillions leurs réactions afin d'éclairer les 
suites à être données, sous forme d'accompagnement pédagogique et technologique, par les membres de l'ÉRI à partir du lieu de la salle de visioconférence ouverte sur le temps de classe, les midis et après l'école.

\subsection{Niveau 2 : Les apprentissages des enseignants}

À ce deuxième niveau, les apprentissages faits par les enseignants à la suite des formations offertes sont, en autant que faire se peut, évalués - à savoir habiletés, savoirs et attitudes. Dans le cas de l'ÉÉR, une large part $\mathrm{du}$ développement professionnel de l'enseignant se produisait dans l'informel, c'est-à-dire que les enseignants faisaient des apprentissages alors qu'ils progressaient dans la mise en œuvre de l'innovation dans leur propre classe en lien avec au moins une autre classe. De plus, la pédagogie de coélaboration de connaissances promue dans l'ÉÉR offrait une toute nouvelle perspective aux enseignants en matière d'apprentissage chez les élèves puisque ces derniers étaient davantage actifs et prenaient la parole à l'écrit pour comprendre des problèmes complexes.

\subsection{Niveau 3 : Le soutien organisationnel}

À ce niveau sont repérés les éléments de soutien organisationnel offert aux enseignants afin qu'ils parviennent à mettre en œuvre ce qu'ils ont appris. Ainsi, ce niveau permet de dépister si les enseignants sont capables ou non de réinvestir dans leur pratique ce qu'ils ont appris soit à cause de faibles conditions organisationnelles ou du fait qu'ils n'ont pas acquis les connaissances nécessaires. Dans le cas de l'ÉÉR, ce sont les conditions d'innovation d'Ely (Ely, 1990) qui ont guidé notre observation de ce qui se passait à ce niveau, surtout en ce qui concernait la disponibilité de temps, la disponibilité de ressources et l'engagement des dirigeants. ${ }^{3}$ Ainsi, pour voir une transformation dans la pratique des enseignants, encore faut-il leur fournir les outils nécessaires et le soutien pour y parvenir.

\subsection{Niveau 4 : L'utilisation des savoirs nouvellement acquis par les enseignants}

À ce quatrième niveau, il s'agit d'analyser si les participants utilisent leurs nouveaux acquis, dans notre cas, une pédagogie de coélaboration de connaissances et les outils technologiques dans le but d'enrichir l'environnement d'apprentissage des élèves. C'est l'observation des pratiques qui permet de voir s'il existe une cohérence entre ce qui est appris et ce qui est fait en classe. Dans l'ÉÉR, il était possible d'avoir accès à plusieurs activités vécues dans les classes, et à une forte majorité des activités vécues en réseau. Ce niveau est souvent très difficile à évaluer sans « faire 
peur » aux enseignants (Guskey, 2003). Dans notre cas, les enseignants ont rendu visible leur pratique enseignante par les traces numériques laissées au sein même des outils utilisés. Il faut aussi savoir que nous avons pu les observer dans l'animation d'activités d'apprentissage en classe à l'aide de la visioconférence.

\subsection{Niveau 5 : Les résultats des élèves}

Le dernier niveau nous ramène à un des aspects essentiels en éducation, c'est-à-dire les résultats observés chez les élèves. Ainsi, le développement professionnel des enseignants s'apprécie-t-il en termes de changements dans l'apprentissage des élèves, soit dans les activités vécues en classe et leurs résultats? Dans l'ÉÉR, tout comme pour les pratiques enseignantes, nous avons pu examiner de près la transformation de l'environnement d'apprentissage de même que les apprentissages réalisés par les élèves afin de mieux cerner comment était appris ce qui était appris.

\section{Méthodologie}

Notre étude a débuté en 2002 alors que l'initiative de l'ÉÉR elle-même commençait. La méthodologie est de type expérimentation de devis (Collins et al., 2004) C'est-à-dire que l'ERI a travaillé de manière proximale avec l'ensemble des intervenants pour concevoir et mettre en œuvre le modèle de l'École éloignée en réseau. Ainsi, dans une dynamique itérative, les équipes-écoles locales, sises dans les différentes commissions scolaires, recevaient des données, analyses et rapports de recherche dont elles discutaient avec les chercheurs afin de mieux comprendre ce qui se passait et ainsi améliorer la mise en œuvre de l'ÉÉR dans leur communauté. L'expérimentation de devis permet d'accélérer la mise en œuvre d'une innovation puisque les chercheurs sont actifs auprès des participants et leur fournissent rapidement et de manière évolutive des résultats de recherche auxquels ils s'intéressent (DRBC, 2002). Les résultats présentés dans cet article se concentrent sur les pratiques enseignantes les plus avancées dans l'ÉÉR, tout en décrivant aussi les pratiques des enseignants débutants. Nous nous concentrons sur les quatre premières phases de l'ÉÉR soit de 2002 à 2010. Le tableau 1 les présente et indique le nombre d'enseignants qui y ont participé. La participation à l'ÉÉR n'était pas aléatoire puisque les écoles étaient choisies par le ministère de l'Éducation en fonction de leur éloignement d'un centre urbain et de leurs problématiques en matière de réussite scolaire. Par contre, chaque enseignant d'une école choisie pouvait décider de participer de manière volontaire à l'ÉÉR. 
C. HAMEL, T. LAFFERIÈRE, S. TURCOTTE , S.ALLAIRE

\begin{tabular}{|l|l|l|}
\hline Phase & Période & $\begin{array}{l}\text { Nombre } \\
\text { d'enseignants }\end{array}$ \\
\hline $\begin{array}{l}\text { Phase I } \\
\text { Mise en place de pro- } \\
\text { jets pilotes }\end{array}$ & $2002-2004$ & 12 \\
\hline $\begin{array}{l}\text { Phase II } \\
\text { Expansion du réseau }\end{array}$ & $2004-2006$ & 118 \\
\hline $\begin{array}{l}\text { Phase III } \\
\text { Consolidation du } \\
\text { réseau }\end{array}$ & $2006-2008$ & 206 \\
\hline $\begin{array}{l}\text { Phase IV } \\
\text { Accélération de } \\
\text { l'innovation }\end{array}$ & $2008-2010$ & 193 \\
\hline
\end{tabular}

Tableau 1 • Les phases de l'École éloignée en réseau et le nombre d'enseignants impliqués

À chacune des phases de l'innovation ÉÉR, l'accroissement de la capacité de certains enseignants permettait d'aller plus loin dans les objets de développement professionnel proposés par le dispositif. Chaque objet de développement professionnel visait à enrichir l'environnement d'apprentissage des classes en augmentant la quantité et la qualité des interactions. Si les objets se sont complexifiés au fur et à mesure que la mise en œuvre progressait, plusieurs objets sont demeurés présents à toutes les phases puisque les enseignants qui travaillaient dans les petites écoles rurales ne demeuraient pas longtemps dans celles-ci et qu'il y avait donc un fort mouvement de personnel. Le tableau 2 présente les principaux objets qui se sont inscrits dans le dispositif de développement professionnel des participants à l'ÉÉR.

\begin{tabular}{|l|l|}
\hline & $\begin{array}{l}\text { Principaux objets de développement profession- } \\
\text { nel }\end{array}$ \\
\hline $\begin{array}{l}\text { Phase I } \\
\text { jets pilotes place de pro- }\end{array}$ & $\begin{array}{l}\text { Deux outils technologiques (visioconférence et KF) } \\
\text { L'élève au centre de son apprentissage (interagir } \\
\text { pour apprendre) } \\
\text { Principes de coélaboration de connaissances } \\
\text { Liens entre ÉÉR et le nouveau curriculum }\end{array}$ \\
\hline Phase II & $\begin{array}{l}\text { Deux outils technologiques (visioconférence et KF) } \\
\text { Principes de coélaboration de connaissances }\end{array}$ \\
& Idées authentiques, problèmes réels \\
& Liens entre ÉÉR et le nouveau curriculum \\
& L'investigation scientifique collaborative \\
\hline Phase III & Deux outils technologiques (visioconférence et KF) \\
Consolidation du & L'amélioration des idées soutenue par un usage mix- \\
réseau & te des deux outils \\
& Principes de coélaboration de connaissances \\
& Idées authentiques, problèmes réels \\
& Évaluation simultanée et transformative \\
\hline
\end{tabular}




\begin{tabular}{|l|l|}
\hline & $\begin{array}{l}\text { Le vocabulaire et le lexique développés par les élèves } \\
\text { Gestion de la classe multiâge en réseau }\end{array}$ \\
\hline Phase IV & Deux outils technologiques (visioconférence et KF) \\
Accélération de & L'amélioration des idées soutenue par l'usage mixte \\
l'innovation & des deux outils \\
& L'utilisation de plugiciels (outils d'analyse des traces \\
& écrites) \\
& Principes de coélaboration de connaissances \\
& Idées authentiques, problèmes réels \\
& Évaluation simultanée et transformative \\
& Soutenir la capacité d'explication des élèves \\
& Effectuer le suivi de la progression du discours des \\
& élèves \\
\hline
\end{tabular}

Tableau 2 - Les principaux objets de développement professionnel dans l'École éloignée en réseau

\subsection{Les outils de collecte de données}

Les outils de collecte de données ont varié tout au long de la mise en œuvre de l'innovation. Les outils de collecte de données portaient sur les apprentissages réalisés par les enseignants à chaque phase de la mise en œuvre de l'ÉÉR. Le tableau 3 résume les différents outils de collecte de données en relation avec les niveaux de Guskey.

\subsubsection{Les entrevues semi-dirigées}

Elles ont été réalisées avec au moins 30 \% des enseignants participants à toutes les phases $(n=92)$ et les enseignants étaient sélectionnés en fonction de leurs années d'expérience dans l'ÉÉR et de la fréquence des activités réalisées en réseau. La fréquence comportait trois degrés soit 1) faible (1 à 3 activités), 2) moyenne (3 à 5 activités) et 3 ) forte (5 activités et plus). L'ensemble des entrevues a été analysé en fonction d'une catégorisation émergente (Krippendorff, 2004) et de l'outil TAMS analyser.

\subsubsection{Le questionnaire en ligne}

Cet instrument s'adressait aux enseignants participants qui y répondaient de manière volontaire. Bon an mal an, une moyenne de $71 \%$ des enseignants inscrits dans l'ÉÉR répondait à ce questionnaire en ligne. Le questionnaire portait sur les compétences, les habiletés et les savoirs des enseignants, sur les conditions d'innovation présentes dans leur contexte de même que sur les retombées de l'ÉÉR dans leur classe multiâge et dans leur pratique. Ce questionnaire était constitué d'une échelle de Likert à cinq niveaux, à laquelle des questions ouvertes ont été parfois ajoutées pour recueillir des commentaires plus précis des participants à l'une ou l'autre phase de la recherche. 


\subsubsection{L'ethnographie virtuelle}

L'ensemble des activités de développement professionnel juste-àtemps était documenté dans une base de données protégée disponible à l'ensemble de l'équipe de recherche sur Internet ${ }^{4}$. Dans cette base de données, l'ethnographie virtuelle (observations et interactions de l'ÉRI avec les praticiens) était consignée de même que les suivis à effectuer. À partir de cette base de données, nous avons pu codifier l'ensemble des activités de développement professionnel des enseignants en réseau. Nous avons aussi documenté l'ensemble des activités d'apprentissage vécues par les enseignants et les élèves en visioconférence et dans le Knowledge Forum par des observations systématiques consignées dans la même base de données. Ainsi, l'ethnographie virtuelle a permis de mieux comprendre les activités qui se déroulaient en classe (observation des activités d'apprentissage) et de faire le suivi des demandes en matière d'accompagnement et de soutien des enseignants participants (observation des activités de développement professionnel).

\begin{tabular}{|l|l|}
\hline Niveaux & Outils de collecte de données \\
\hline Réactions des participants & $\begin{array}{l}\text { Questionnaire en ligne } \\
\text { Entrevues semi-dirigées }\end{array}$ \\
\hline Apprentissage des enseignants & $\begin{array}{l}\text { Questionnaire en ligne } \\
\text { Entrevues semi-dirigées }\end{array}$ \\
\hline Le soutien organisationnel & $\begin{array}{l}\text { Questionnaire en ligne } \\
\text { Entrevues semi-dirigées }\end{array}$ \\
\hline L'utilisation des savoirs et habile- & $\begin{array}{l}\text { Ethnographie virtuelle } \\
\text { tés par les enseignants }\end{array}$ \\
& Entrevues semi-dirigées \\
& Questionnaire en ligne \\
& Analyse du discours en ligne des enseignants \\
& et des élèves \\
\hline Les résultats au niveau des élèves & Ethnographie virtuelle \\
& Questionnaire sur la motivation (Autodé- \\
& termination) \\
& Test de compréhension de lecture (PIRLS \\
& Analyse du discours en ligne des élèves \\
\hline
\end{tabular}

Tableau 3 • Les outils de collecte de données en fonction des cinq niveaux de Guskey (Guskey, 2003)

\section{Les résultats}

Nous vous présentons, dans le tableau 4, les résultats en relation avec le développement professionnel des enseignants dans l'ÉÉR et les niveaux de Guskey. 
Tableau 4 • Le développement professionnel des enseignants dans l'ÉÉR

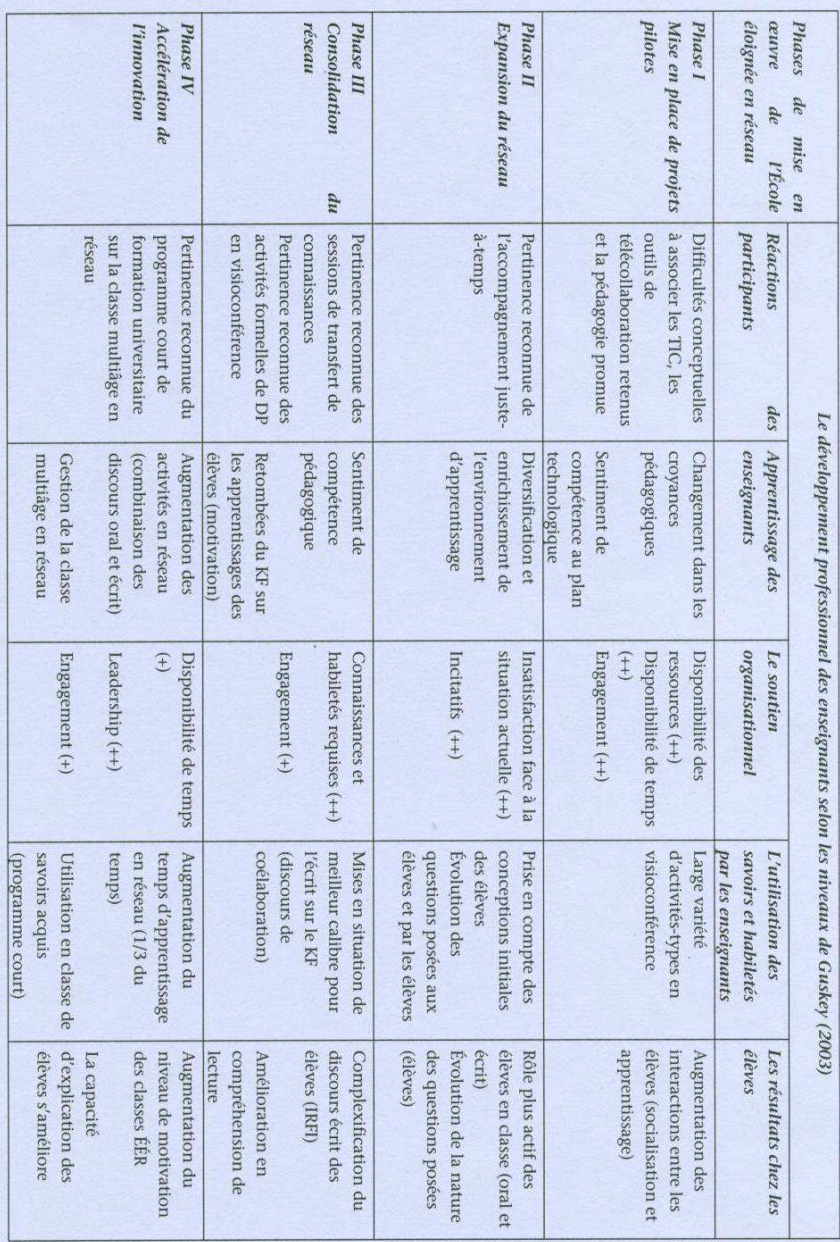




\subsection{La réaction des enseignants: une expérience déséquilibrante, mais transformative}

À la suite des activités de développement professionnel (DP) portant sur les outils de télécollaboration, dans la phase I de la mise en œuvre de l'ÉÉR, les perceptions des enseignants en classe ont été marquées par une certaine difficulté conceptuelle, notamment en ce qui concerne les usages en classe. Dans les entrevues, les enseignants témoignent abondamment de leur difficulté à voir comment ils allaient utiliser en classe les outils (56\% de l'ensemble des unités codées). D'ailleurs, bien que l'Internet à large bande passante et la réforme du curriculum soient maintenant présents partout sur le territoire québécois, cette difficulté conceptuelle demeure encore aujourd'hui chez les enseignants qui débutent dans l'ÉÉR (Laferrière et al., 2011). Une enseignante exprimait ainsi sa réaction à la suite des premières rencontres :

Je me demandais comment j'allais pouvoir utiliser les outils dans la classe. On n'avait même pas l'Internet dans la classe l'année d'avant, c'était une grosse affaire. Et la coélaboration de connaissances, je comprenais que ça allait bien avec la réforme [du curriculum], mais ça aussi on l'avait pas l'année d'avant! (Enseignante 7, juin 2002)

Une enseignante arrivée en phase III exprime ainsi ses premières réactions :

Je me souviens que lorsqu'on m'a expliqué la coélaboration et l'utilisation du KF dans la classe, il y avait plein d'exemples et ça semblait vraiment l'fun, mais c'était tellement loin de ce que je faisais dans ma classe. J'avais toute une côte à monter ! (Enseignante 26, juin 2008)

À cet égard, bien que le dispositif d'accompagnement juste-à-temps (salle de garde en visioconférence) ait été disponible depuis le début de la mise en œuvre, il demeure que c'est en phase II que les enseignants perçoivent explicitement son utilité et sa pertinence dans les entrevues réalisées (49 \% des unités codées traitent de ce point) (Hamel et al., 2012). Un enseignant explique en ces termes la pertinence du développement professionnel offert dans la salle de garde en visioconférence (salle TACT) :

Quand t'es dans l'action et que tu veux que ça marche, ça change les choses. Moi quand j'ai essayé de faire une activité pis que rien ne fonctionnait pour ajouter les échafaudages, j'ai été dans la salle TACT et quelqu'un m'a aidé au moment même. Et quand tu examines ce que les élèves 
ont écrit dans le KF et que la discussion est bloquée, ça aide d'avoir une autre paire d'yeux qui a des idées. (Enseignant 67, juin 2006)

Au fil des phases ÉÉR, la progression dans les connaissances et les habiletés des enseignants s'est répercutée en un développement de capacité important en matière d'usage des outils de télécollaboration et de coélaboration de connaissances dans la classe. Et les enseignants ont perçu que ce sont les sessions de transfert nationales ${ }^{6}$ qui permettaient de bâtir une capacité plus importante encore chez leurs collègues qui débutaient dans l'ÉÉR au moment de la phase III (41 \% des unités de sens codées). En effet, c'est à ce moment que les sessions de transfert sont devenues très ancrées dans le partage de pratiques entre enseignants puisqu'il y avait désormais un bagage important d'activités d'apprentissage diversifiées qui se réalisaient dans les classes.

Finalement, lors de la phase IV, les enseignants ont souvent exprimé leur intérêt à voir leur expertise reconnue en matière de gestion de la classe multiâge en réseau de même que leur souhait que d'autres collègues puissent bénéficier d'une formation plus formelle à ce sujet. L'Université du Québec à Chicoutimi, l'Université du Québec en AbitibiTémiscamingue et l'Université du Québec en Outaouais ont donc développé conjointement un programme court de formation universitaire à ce sujet, programme qui est toujours offert aujourd'hui dans l'ensemble des universités du Québec (réseau UQ) (Allaire et al., 2010)L'apprentissage des enseignants: une complexification de la pratique en classe multiâge en réseau

Si les enseignants avaient au départ une difficulté à concevoir l'utilisation des outils de télécollaboration dans leur classe de même que la coélaboration de connaissances, ils avaient aussi de sérieux doutes quant à la capacité des élèves à être actifs dans leurs apprentissages en visioconférence et sur le KF (autonomie et motivation des élèves à apprendre). Néanmoins, l'analyse de leurs croyances (Ajzen et Fishbein, 1980) a permis d'identifier une évolution dans leur perception des capacités des élèves à cet égard. Nous avons aussi repéré une certaine évolution dans la compréhension des possibilités qu'amène le réseau pour la collaboration à l'extérieur de l'école, de la complémentarité entre la réforme du curriculum et l'ÉÉR et de l'expertise professionnelle particulière qu'ils développaient (Laferrière et al., 2004a). De plus, les enseignants se disaient beaucoup plus compétents au sujet de l'usage des technologies dans leur 
quotidien alors que la plupart d'entre eux se définissaient comme très peu compétents au départ.

Si les activités d'apprentissage ont été fortement concentrées en visioconférence lors de la phase I, elles se sont nettement réparties sur les deux outils de télécollaboration durant la phase II (Allaire et al., 2006a). De fait, les activités écrites dans le KF ont été quatre fois plus nombreuses que lors de la phase précédente, et les enseignants mentionnaient mieux comprendre comment utiliser le KF en lien avec le curriculum. D'ailleurs, un accompagnement pédagogique plus ciblé sur l'apprentissage des sciences et de la technologie démontre que les enseignants font davantage d'activités dans ce domaine et que le KF leur apparait comme un outil facilitant (Turcotte et Hamel, 2011).

Dans la phase III, la pratique enseignante dans le contexte d'une classe ÉÉR continue de se développer et elle se définit clairement dans leur discours comme une pratique dans une classe en réseau et les enseignants perçoivent avoir développé une compétence pédagogique distinctive. Les enseignants mentionnent souvent la coélaboration de connaissances dans leur discours, en lien avec l'utilisation du KF dans leur classe. Ils perçoivent des retombées importantes sur les apprentissages des élèves ce qui les incite à poursuivre leurs apprentissages et à demander encore plus de soutien dans la salle de visioconférence permanente. Ce développement professionnel juste-à-temps est majoritairement axé sur des besoins pédagogiques en lien avec les activités de coélaboration de connaissances. Les demandes de nature technique perdurent, mais elles sont davantage axées sur des fonctions avancées du KF comme le fait d'écrire une note synthèse des propos des camarades (Hamel et al., 2012a).

Cet accroissement de la capacité des enseignants et cette complexification de la pratique en classe ont amené un nouveau positionnement pour mieux définir l'expertise acquise. C'est durant la phase IV que l'expression de gestion de la classe multiâge en réseau devient partie intégrante du modèle (Allaire et al., 2012) puisqu'elle fait partie de l'identité des enseignants. Ainsi, les enseignants en classe multiâge perçoivent que l'ÉÉR est profitable pour agir sur les facteurs qui favorisent la réussite. La quantité et la qualité des activités vécues avec le support des deux outils de télécollaboration continuent de progresser, et ce même si le nombre d'enseignants est presque semblable à la phase III. Les enseignants mentionnent aussi qu'ils sont davantage en mesure de combiner l'utilisation des deux outils (oral et écrit) pour soutenir l'apprentissage des élèves et 
qu'il est essentiel de partir du curriculum pour y parvenir (Laferrière et al., 2011). Finalement, les enseignants qui font le plus de KF en collaboration avec d'autres classes perçoivent des retombées plus importantes sur les apprentissages de leurs élèves que ceux qui en font moins.

\subsection{Un soutien organisationnel essentiel pour la mise en œuvre}

La mise en œuvre de l'ÉÉR est démarrée rappelons-le, à l'instigation du ministère de l'Éducation, et ce sont les dirigeants des commissions scolaires qui ont pris la décision de s'y inscrire. Durant l'ensemble des quatre phases, les conditions d'innovation (Ely, 1990) ont été observées et validées auprès de chaque site afin de mieux comprendre la mise en œuvre (Laferrière et al., 2011). Ainsi, durant la phase I, l'engagement des dirigeants a été une condition non négligeable de la réussite puisque ce sont eux qui ont accompagné les équipes-écoles et qui ont assuré un suivi auprès de l'équipe de recherche-intervention lors des itérations ${ }^{8}$. Ce sont eux qui se sont assurés que les enseignants aient des conditions favorables à l'innovation, notamment en octroyant des ressources importantes pour y arriver. Par exemple, un conseiller pédagogique a été libéré afin d'accompagner les enseignants dans la plupart des sites et les techniciens étaient toujours disponibles afin d'assurer la viabilité technologique de l'ÉÉR. De plus, les enseignants ont eu du temps reconnu dans leur tâche afin de planifier avec leurs collègues, mais aussi de participer aux activités de recherche. Toutes ces conditions n'ont pas été mises en œuvre si aisément, notamment les conditions technologiques, puisque plusieurs critères de sécurité technologiques prenaient le dessus sur les besoins pédagogiques. Ce sont donc les trois conditions qui ont été déterminantes au début de l'initiative.

$\mathrm{Au}$ cours de la phase II, les enseignants connaissaient davantage les possibilités que leur offrait l'ÉÉR et c'est l'insatisfaction face à la situation actuelle qui a joué un rôle important. En effet, c'est l'isolement professionnel vécu par les enseignants de très petites écoles qui a amplifié leur besoin de collaborer avec d'autres collègues et ils ont utilisé les outils pour en arriver à se sentir moins isolés dans leur pratique qu'ils trouvaient très complexe compte tenu du peu de ressources et du nombre de niveaux dans leur classe (Turcotte et Hamel, 2008). De plus, les enseignants ressentaient une insatisfaction importante quant à la motivation à apprendre de leurs élèves et ils désiraient trouver des moyens pour améliorer leur réussite scolaire. Comme les enseignants avaient les mêmes élèves plusieurs 
années consécutives, ils cherchaient des moyens de diversifier l'environnement d'apprentissage (Allaire et al., 2006a). La phase II correspond aussi au moment d'expansion du réseau, ce qui a amené de plus grandes possibilités de collaboration entre différents milieux. Elle a été caractérisée par des incitatifs offerts aux enseignants, tout comme en phase I, notamment l'octroi d'un ordinateur portatif par enseignant, mais aussi d'ordinateurs dans la classe afin que les élèves puissent travailler à partir de là, plutôt que dans des laboratoires informatiques. D'autres incitatifs se sont ajoutés, entre autres, la participation à un colloque international sur la coélaboration de connaissances pour les enseignants plus avancés.

C'est en phase III que les résultats de recherche montrent que les connaissances et habiletés des enseignants ont atteint un nouveau niveau, notamment parce qu'un plus grand nombre d'enseignants n'étaient pas des débutants dans le modèle de l'ÉÉR (62\% des enseignants ont déjà fait des activités ÉÉR dans leur classe). D'ailleurs, il devient plus clair que les enseignants qui font le plus d'activités dans l'ÉÉR ne sont pas des enseignants débutants, mais des enseignants qui enseignent depuis plus de trois ans. Il existe donc maintenant une masse critique d'enseignants qui font de la coélaboration de connaissances en collaboration à l'aide d'outils technologiques. C'est dans cette phase que l'importance de l'engagement des dirigeants est remise à l'avant-plan puisque plusieurs sites ont connu un mouvement de personnel important et un nouveau travail de démarrage est nécessaire pour assurer le maintien de l'ÉÉR. En effet, plusieurs conditions d'innovation sont tenues pour acquises alors que les besoins des enseignants se modifient et que le soutien demeure nécessaire.

Dans la phase IV, l'engagement des dirigeants a été très sollicité en cette phase d'accélération de l'innovation dans quatre sites qui présentent d'excellentes conditions pour assurer la pérennité de l'innovation. Plusieurs nouveaux enseignants se sont joints aux équipes déjà en place et la condition de la disponibilité de temps est apparue de nouveau comme primordiale puisque les enseignants avaient augmenté leur temps d'activités en réseau jusqu'à une heure par jour. De plus, c'est au cours de cette phase que le leadership exercé pour la mise en œuvre de l'ÉÉR se distribue davantage dans les commissions scolaires et qu'il n'est plus l'apanage uniquement des dirigeants, mais aussi celui des enseignants et des directions d'établissement (Hamel, 2011). C'est d'ailleurs à partir de cette phase que l'institutionnalisation du modèle de l'ÉÉR est envisagée 
par les instances du ministère de l'Éducation du Québec étant donné les résultats obtenus chez les enseignants et les élèves.

\subsection{L'utilisation du savoir et des habiletés nouvellement acquis par les enseignants : une visibilité du cœur de l'action en classe}

Lors de la phase I, l'attrait de la visioconférence en classe est important pour les enseignants et les élèves et un grand nombre d'activités sont réalisées grâce à cet outil. Une variété importante d'activités en visioconférence ont été repérées (19 au total) et celles-ci sont en lien avec les principaux volets de l'ÉÉR dont l'apprentissage des élèves, le développement professionnel des enseignants, l'administration de l'innovation et les besoins techniques. Ces activités-types ont continué à être présentes et à augmenter durant les trois autres phases de l'ÉÉR avec une prépondérance importante pour les activités techniques au début de chaque phase puisque de nouveaux enseignants s'ajoutaient et que certaines configurations technologiques étaient à reprendre.

\begin{tabular}{|c|c|}
\hline \multirow{3}{*}{ Administration } & $\begin{array}{l}\text { Définition des rôles et attribution des fonctions } \\
\text { Établissement et maintien de contacts et de parte- } \\
\text { nariats }\end{array}$ \\
\hline & Recherche \\
\hline & Réseautage des acteurs \\
\hline \multirow{2}{*}{ Technique } & Reconstitution de problèmes techniques \\
\hline & Résolution spontanée de problèmes techniques \\
\hline \multirow{6}{*}{$\begin{array}{l}\text { Développement } \\
\text { professionnel }\end{array}$} & Veille technoémotionnelle \\
\hline & Mise en route technologique \\
\hline & Garde virtuelle \\
\hline & Accompagnement pédagogique \\
\hline & $\begin{array}{l}\text { Planification et coordination d'activités pédagogi- } \\
\text { ques }\end{array}$ \\
\hline & Retour sur l'expérience \\
\hline \multirow{7}{*}{$\begin{array}{l}\text { Apprentissage } \\
\text { d'élèves }\end{array}$} & Travail en équipe d'élèves délocalisée \\
\hline & Mise à contribution par l'élève de son expertise \\
\hline & Leçons en classe élargie \\
\hline & Mentorat \\
\hline & Mini-profs \\
\hline & Team teaching délocalisé \\
\hline & Encadrement par un adulte autre que l'enseignant \\
\hline
\end{tabular}

Tableau 5 • Les activités-types en visioconférence 
Lors de la phase II, les objets de développement professionnel ont été davantage centrés sur la coélaboration de connaissances, entre autres, partant du principe problèmes authentiques et idées réelles. L'analyse des questions formulées par les enseignants aux élèves dans le KF, après discussion en classe ou pas, démontre une progression dans le nombre de questions ouvertes posées, c'est-à-dire des questions centrées sur l'interprétation et l'explication ( $1 / 3$ des questions sont ouvertes) (Allaire et al., 2006b). Toutefois, les activités de développement professionnel centrées sur l'investigation scientifique en sciences montrent que les enseignants ne parviennent pas à compléter l'ensemble du processus d'investigation avec leurs élèves. Néanmoins, ils sont maintenant capables de tenir compte des conceptions initiales des élèves et ils consacrent davantage de temps à l'enseignement des sciences au primaire (Turcotte, 2008).

La progression de l'application du principe problèmes authentiques et idées réelles se constate par le nombre grandissant de questions posées par les élèves dans le KF, et suscite une analyse pointue, partant de la grille de Hmelo-Silver et Barrows (Hmelo-Silver et Barrows, 2008) de la nature des questions posées aux élèves et par les élèves dans le KF. Il est mis en évidence que quatre questions sur cinq appellent des réponses longues et complexes que ce soit les élèves ou les enseignants qui les posent. Les activités réalisées autant dans le KF qu'en visioconférence partent de problèmes plus complexes et elles sont de meilleur calibre (Laferrière et al., 2008). De plus, alors que le discours présent dans le KF était davantage un discours centré sur la consignation d'information, le discours écrit connait une progression pour être davantage un discours de coélaboration de connaissances ( $46 \%)$.

Finalement, en phase IV, un grand nombre d'enseignants s'estiment capables de réaliser un plus grand nombre d'activités en réseau par l'usage des deux outils. Les enseignants les moins expérimentés dans l'ÉÉR (moins d'un an) passent moins de temps à réaliser des activités en réseau, mais le temps consacré à l'ÉÉR augmente de manière importante à mesure que l'enseignant acquiert de l'expérience. Quelques enseignants deviennent des modèles pour leurs collègues lorsqu'ils mentionnent qu'ils ont mis explicitement dans leur horaire le temps de travail en réseau, à raison d'une heure par jour. La figure 1 illustre cette analyse. 
Figure 1 • Part du temps consacré mensuellement à l'ÉÉR en fonction du

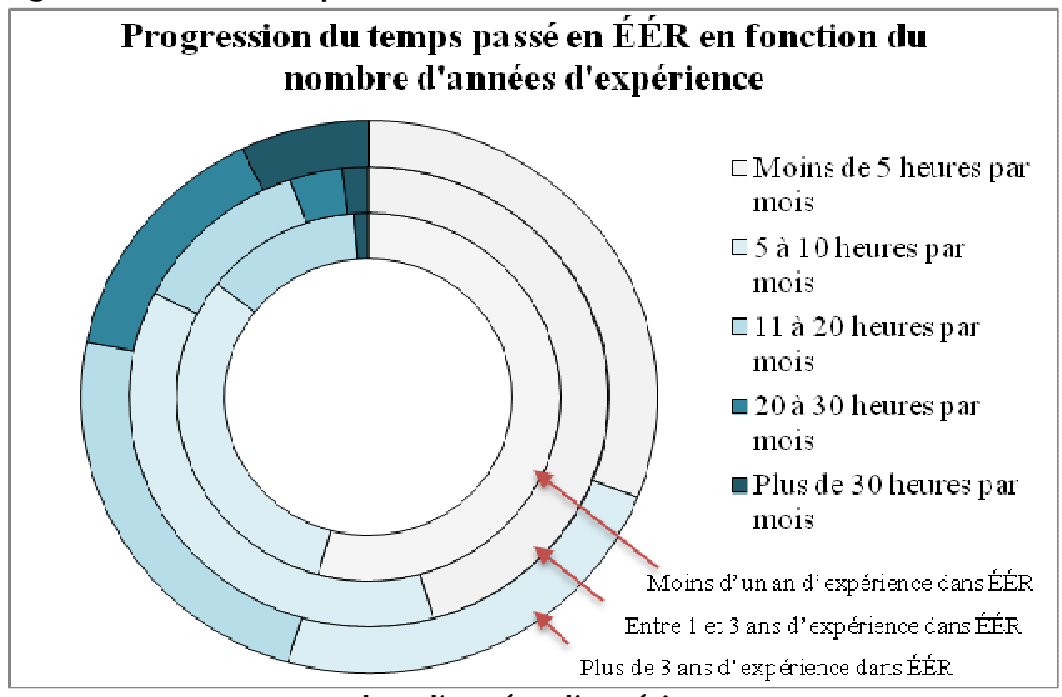

nombre d'années d'expérience

De plus, l'analyse du discours des enseignants qui ont fait le programme court de formation universitaire sur la classe multiâge en réseau montre un réinvestissement des concepts du cours dans la pratique enseignante et dans le discours des enseignants. De plus, les enseignants apprécient pouvoir travailler en coélaboration de connaissances dans le cours, ce qui leur permet une transposition théorie pratique.

\subsection{Les résultats des élèves: des interactions centrées sur les élèves}

Tout ce dispositif de développement professionnel visait à enrichir l'environnement d'apprentissage des élèves par l'usage des TIC pour augmenter les interactions à des fins d'apprentissage. Dans la phase I, il s'agit d'un résultat important puisque les analyses de l'activité en classe et de l'activité en réseau montrent que l'activité en réseau amène une transformation puisque ce sont les élèves qui interagissent avec le support des outils de télécollaboration et qu'ils ont davantage de temps de parole en classe. La figure 2 illustre ce résultat. Dans les activités en visioconférence, c'est le travail en équipe délocalisée qui occupe une forte proportion du temps (46\%). Ainsi, les élèves travaillent ensemble à faire des mathématiques, du français, ils préparent des expériences scientifiques, ils construisent une compréhension d'un texte qu'ils ont lu, ils interprètent une 
œuvre. Bref, ils interagissent pour apprendre et ils socialisent, deux missions importantes de l'école québécoise.

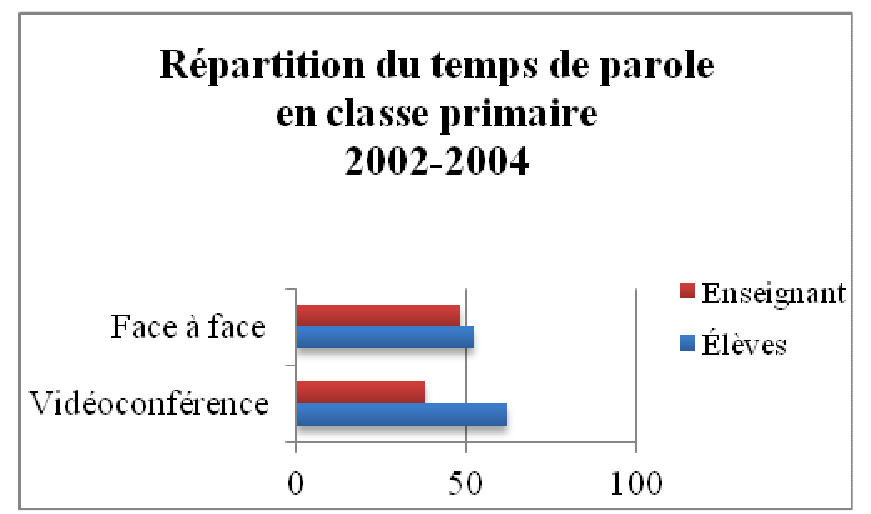

Figure 2 - Répartition du temps de parole en classe au primaire (Phase I)

Dans la phase II, il y a un passage important des activités réalisées en visioconférence, aux activités écrites dans le KF. Probablement par un effet de modelage des enseignants, les élèves ont posé des questions ouvertes dans le $\mathrm{KF}$ et ils ont démontré une capacité à coélaborer des connaissances lorsque les questions étaient ouvertes. Les élèves ont aussi témoigné de leur appréciation de l'ÉÉR dans la classe puisqu'elle leur permettait de mieux communiquer oralement, de comprendre de "gros problèmes importants » et de travailler plus fort pour se faire comprendre à l'écrit parce que de vraies personnes (destinataires authentiques) les lisaient. De plus, avec de l'aide, les élèves sont en mesure d'utiliser les échafaudages de l'investigation scientifique de manière cohérente. Les enseignants témoignent utiliser maintenant les stratégies de questionnement dans leur enseignement courant et pas seulement en réseau.

Dans la phase III, le discours écrit des élèves se complexifie pour en arriver à ne pas se centrer uniquement sur les conceptions initiales d'un problème à l'étude, mais aussi aller vers de nouveaux questionnements (Laferrière et Lamon, 2010). La structure de discours dans le KF n'est donc plus centrée sur l'I-R-E (Initiative-Réponse-Évaluation) (Cazden, 2001), mais plutôt sur un troisième tour de parole pris par l'élève pour amener un nouveau questionnement. De plus, le test de compréhension écrite (PISA), soumis à chaque année aux élèves de $4^{\mathrm{e}}$ année, démontre une amélioration des résultats des élèves ÉÉR par rapport aux groupes contrôles. Les élèves des classes ÉÉR obtenaient des résultats plus faibles et ils arrivent maintenant à rattraper leurs pairs des classes contrôles'. Il ne 
s'agit pas d'un effet direct de l'ÉÉR puisque plusieurs autres facteurs ont pu influencer leur compétence en compréhension de texte. Toutefois, il s'agit d'un résultat pour nous qui témoigne d'une amélioration de l'environnement d'apprentissage des élèves.

En phase IV, c'est la capacité d'explication des élèves qui a été étudiée, afin de mieux comprendre l'apport de l'écriture dans le KF. Ainsi, les élèves ont amélioré leur capacité d'explication à l'oral à propos des phénomènes étudiés quand ils ont écrit des notes de meilleure qualité dans le KF et quand ils ont davantage lu dans le KF. Les élèves du groupe contrôle qui n'ont pas du tout utilisé le KF n'arrivent pas à rattraper les élèves qui ont fait du KF dans leur capacité d'explication à l'oral. Au niveau de la motivation des élèves, celle des élèves qui fréquentaient une école ÉÉR en 2002 était significativement inférieure à celle des élèves des groupes contrôles. Toutefois, le niveau de motivation des élèves des écoles ÉÉR a rattrapé (2008) puis dépassé (2009) le niveau de motivation des élèves des écoles non ÉÉR. Comme pour les résultats en compréhension de lecture, il ne s'agit pas d'un effet direct de l'ÉÉR, mais d'un résultat qui témoigne de l'enrichissement de l'environnement d'apprentissage, l'ÉÉR en faisant partie. La figure 2 présente un nuage du discours des élèves quand ils répondent à la question : C'est quoi être dans une classe ÉÉR? On remarque un accent marqué sur les savoirs (connaissances) et les savoir-faire (faire, fait), sur l'enrichissement (plus) ainsi que la présence de la dimension collective de l'apprentissage (ensemble, ils, autres).

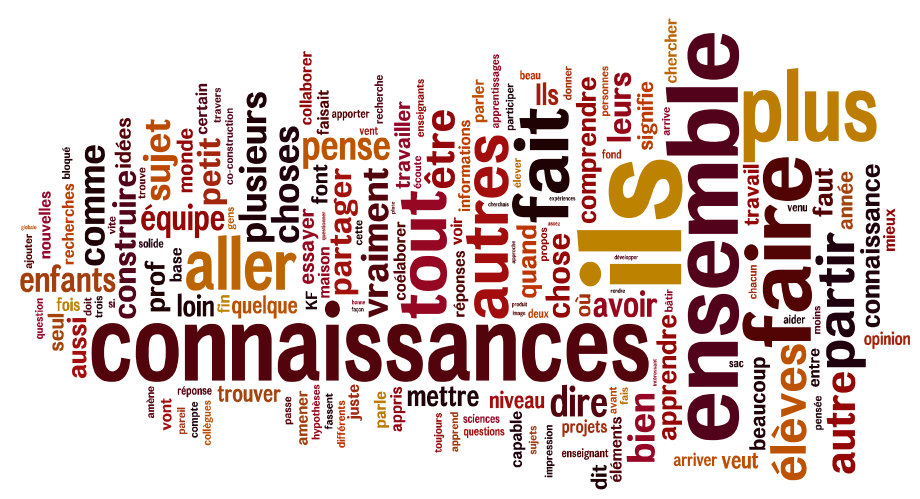

Figure 3 • Nuage de mots des élèves à propos de l'ÉÉR 


\section{Discussion}

\subsection{Un dispositif de développement professionnel fondé sur des résultats de recherche}

Les résultats présentés dans cet article témoignent de la vigueur de la recherche et de l'intervention effectuées dans les écoles de l'ÉÉR durant les huit années rapportées. Ces résultats étaient réinvestis rapidement lors de chacune des phases auprès des enseignants et des autres intervenants grâce au processus itératif présent dans l'expérimentation de devis (Collins et al., 2004). Ainsi, tant l'analyse des conditions d'innovation présentes, l'analyse du discours oral et écrit des élèves, les résultats aux tests de motivation et de compréhension écrite étaient retournés rapidement dans les sites participants afin de servir d'objet de réflexion aux acteurs. Il n'était pas toujours facile de recevoir ces résultats, surtout au début de l'initiative et cela créait parfois une tension entre les différents acteurs. Certains avaient l'impression de recevoir leur «bulletin». Par exemple, lorsque la condition d'innovation sur la disponibilité des ressources mettait en évidence que les enseignants étaient prêts à utiliser les outils dans leur classe et que les dispositifs de sécurité informatique les en empêchaient, il fallait en arriver à résoudre ce problème tout en préservant les besoins liés à la sécurité. Pour les chercheurs, il a aussi fallu utiliser les résultats à bon escient afin de préserver l'anonymat des enseignants et les résultats de leur classe pour en arriver à une analyse plus globale de l'action dans une classe ÉÉR. Il demeure que, pour l'ensemble des intervenants, le processus itératif de recherche-intervention soutenu par un comité de suivi local a permis d'appuyer l'ensemble du processus d'innovation et la prise de décisions (Hamel, 2011). Le design de la recherche a aussi été construit en collaboration avec les praticiens au fur et à mesure que la pratique en classe se complexifiait. Ainsi, ce sont les enseignants qui ont demandé à ce que les chercheurs étudient la capacité d'explication des élèves afin de valider leurs perceptions sur l'utilisation $\mathrm{du} \mathrm{KF}$ et sur les apprentissages des élèves (Turcotte et al., 2011). Aujourd'hui encore, le dispositif de soutien juste-à-temps est utilisé quotidiennement par les enseignants pour répondre à des besoins divers en lien avec la mise en œuvre de l'ÉÉR dans leur classe. Leur questionnement suit aussi les courants puisqu'actuellement plusieurs d'entre eux ont accès à des technologies mobiles et ils veulent les utiliser de manière collaborative avec les outils de l'ÉÉR. 


\subsection{Le processus de coélaboration de connaissances: une théorie qui appuie le dispositif de développement professionnel}

Tout comme le veut le processus de coélaboration de connaissances, notre dispositif de développement professionnel, parce qu'il est en contexte d'innovation, a impliqué des acteurs disposés à travailler ensemble sur des problèmes réels et authentiques : inventer l'École éloignée en réseau afin d'enrichir l'environnement d'apprentissage des élèves. Ainsi, les enseignants comme les chercheurs ont mis en commun leurs idées et créé une nouvelle façon de "faire l'école », en prenant en considération les contraintes et motivations de chacun. D'un côté, les chercheurs puisaient aux pratiques exemplaires des milieux qu'ils connaissaient ou dont ils avaient lu les résultats de recherche et de l'autre, les enseignants adaptaient leur réalité aux activités rendues possibles par les nouveaux outils s'offrant à eux. Parce que les uns comme les autres gagnaient à enrichir leurs propres pratiques par ce processus, et partageaient le même objectif d'améliorer l'expérience scolaire des élèves, ils ont peu à peu construit une vision partagée de cette nouvelle école en réseau.

De même, c'est l'amélioration du savoir collectif qui a été le moteur de l'innovation, notamment en ce qui concerne les pratiques des enseignants à l'aide des outils de télécollaboration. Chaque expérience enrichissante, comme chaque écueil relevé sont partagés au sein de la communauté de l'ÉÉR : les sessions de transfert, par exemple, sont chaque année un moment phare pour les enseignants impliqués puisque c'est le moment pour chacun d'exposer devant ses pairs le fruit de ses efforts. En mettant ainsi de l'avant leurs apprentissages comme leurs difficultés, les enseignants comme les chercheurs visent à trouver les meilleures façons d'avancer, d'un projet à l'autre. Si, par exemple, des classes ont réalisé qu'il valait mieux, pour maintenir la motivation des élèves, concentrer sur deux semaines leurs activités scientifiques en réseau, l'information est partagée à d'autres classes qui sont en train de planifier leurs activités. Et si de nombreuses classes ont travaillé ensemble à un projet commun ambitieux, leurs enseignants partageront d'emblée les façons d'organiser leur travail pour que cela soit le plus profitable. C'est dire que plusieurs apprentissages réalisés et connaissances développées par les pratiques des enseignants dans le dispositif ont été réinvestis auprès des collègues.

L'un des principes de coélaboration de connaissances est de faire de la classe un lieu de démocratisation du savoir et L'École éloignée en réseau 
se veut un lieu de démocratisation du savoir. Ainsi, peu importe où se trouve l'école d'un élève au Québec, il doit avoir accès aux mêmes chances de succès et à la même qualité d'éducation. Au départ, les enseignants ne savaient quoi penser des chercheurs qui arrivaient dans leur région, flanqués des gens du ministère de l'Éducation pour discuter d'une école à inventer et d'Internet à large bande passante. Malgré l'intention clairement énoncée par ceux-ci de s'arrimer aux besoins des différentes classes, les appréhensions des enseignants et des autres intervenants étaient présentes d'une commission scolaire à l'autre. La réforme du curriculum n'avait pas été accueillie chaudement partout et certains esprits se méfiaient d'une nouvelle initiative ministérielle. Mais au contact de l'équipe de recherche-intervention, qui rappelait que tout était à inventer, qui tâchait de ne pas imposer ses idées et qui se plaçait au service des enseignants, les enseignants ont pu prendre leur place dans l'ÉÉR et participer à sa création.

\section{Conclusion}

Plus que tout, ce retour sur d'importants résultats de l'ÉÉR nous rappelle que ce qui semblait le plus attrayant pour les enseignants lors des démarrages, soit d'avoir accès à de nouvelles personnes, et donc, à de nouvelles idées, demeure à ce jour l'aspect le plus porteur de l'initiative. L'isolement professionnel des enseignants des écoles rurales avait largement été sous-estimé, plus encore dans un contexte de réforme du curriculum. Comme le vise la pédagogie de la coélaboration de connaissances, l'ÉÉR permet d'ouvrir la classe à une diversité de nouvelles idées, des idées à améliorer est-il répété. D'une toute petite école, peu importe où qu'elle soit sur le territoire, il devient possible d'en joindre d'autres, non seulement pour y puiser des informations, mais d'abord pour y vivre de nouvelles interactions et partager avec d'autres des idées afin d'en produire de meilleures. Le dispositif de développement professionnel mis en place constitue une des raisons de son succès et de sa continuité dans le système d'éducation au Québec. En combinant le soutien par les outils de télécollaboration et la pression des résultats de recherche, les pratiques enseignantes ont permis d'offrir un plus grand nombre d'interactions à des fins d'apprentissage pour les élèves et, par le fait même, d'enrichir l'environnement d'apprentissage de la petite école rurale.

\section{BIBLIOGRAPHIE}

AJZEN I., FISHBEIN M. (1980). Understanding attitudes and predicting social behavior. Englewood Cliffs, NJ: Prentice Hall. 
ALLAIRE S. (2007). Use of a lexicon applet for the evaluation of students' understanding of science concepts : The Case of the Quebec Remote Networked Schools (RNS). Toronto: The Ontario Institute for Studies in Education.

ALLAIRE S., BEAUDOIN J., BREUlEUX A., HAMEL C., TURCOTTE S. (2006). L'École éloignée en réseau (ÉÉR) Rapport final (Phase 2) (p. 124).

ALLAIRE S., HAMEL C., C.-BOUFFARD M.-H., LAFERRIÈRE T. (2006). The emergence of the principle «Real ideas, authentic problems» in teachers' and students' usage of Knowledge Forum in remote networked schools. Toronto : Knowledge Building Summer Institute (OISE).

ALLAIRE S., HAMEL C., GAUDREAULT-PERRON J., \& LAFERRIÈRE T. (2012). L'apprentissage collaboratif en réseau au profit de l'intervention en classe multiâge. Revue de recherche en éducation, Vol. 2, p. 1-16.

ALLAIRE S., PELlERIN G., BEAUdOIN M., COUTURE C., TURCOTTE S. (2010). Développement d'un programme de formation interuniversitaire en réseau : pallier une situation découlant des mouvements démographiques au Québec. In Journées Communication et Apprentissage Instrumentés en Réseau (JOCAIR 2010). Amiens.

BORKO H. (2004). Professional development and teacher learning: Mapping the terrain. Educational Researcher, Vol. $33 \mathrm{n}^{\circ} 8$, p. 3-15. doi:10.3102/0013189X033008003

CAZDEN C. B. (2001). The language of teaching and learning. NH: Heinemann.

COLLINS A., JOSEPH D., BIELACZYC K. (2004). Design research: Theoretical and methodological Issues. The Journal of the Learning Sciences, Vol. $13 \mathrm{n}^{\circ} 1, \mathrm{p} .15-$ 42. doi:10.1207/s15327809jls1301_2

COLLINSON V., KOZINA E., KATE LIN Y.-H., LING L., MATHESON I., NEWCOMBE L., ZOGLA I. (2009). Professional development for teachers: a world of change. European Journal of Teacher Education, Vol. $32 \mathrm{n}^{\circ} 1$, p. 3-19. doi:10.1080/02619760802553022

DBRC. (2002). Design-based research: An emerging paradigm for educational inquiry. Educational Research, Vol. $32 \mathrm{n}^{\circ} 1$, p. 5-8.

ELY D. (1990). Conditions that facilitate the implementation of educational technology innovations. Journal of Research on Computing in Education, Vol. $23 \mathrm{n}^{\circ} 2$, p. 298-305.

FLORES M. A. (2005). How do teachers learn in the workplace ? Findings from an empirical. Journal of In-Service Education, Vol. 31 n $^{\circ} 3$, p.485-508.

FULLAN M. (1995). The limits and the potential of professional development. In T. R. Guskey \& M. Huberman (Eds.), Professional Development in Education: New Paradigms and Practices (pp. 253-267). New York, NY: Teacher College Press.

GARET M., PORTER A., DESIMONE L., BIRMANE B. F., YOON K. S. (2001). What makes professional development effective? Results from a national sample of teachers. American Educational Research Journal, Vol. $38 n^{\circ} 4$, p. 915-945. Disponible sur internet : http://aer.sagepub.com/content/38/4/915.short

GODERYA-SHAIKH F. (2010). Professional development with follow-up for an effective paradigm shift. International Journal for Cross-Disciplinary Subjects in Education (IJCDSE), Vol. $1 \mathrm{n}^{\circ} 3$, p. 182-189. Disponible sur internet : http://www.infonomics-society.org/IJCDSE/Professional Development with Followup for an Effective Paradigm Shift.pdf

GUSKEY T. R. (2002). Professional development and teacher change. Teachers and Teaching: theory and practice, Vol. $8 \mathrm{n}^{\circ} 3, \mathrm{p} .381-391$. Disponible sur internet : http://www.tandfonline.com/doi/full/10.1080/135406002100000512 
GUSKEY T. R. (2003). What makes professional development effective? . Phi Delta Kappan , Vol. $84 \mathrm{n}^{\circ} 10$, p. 748-750. Disponible sur internet : http://www.kappanmagazine.org/content/84/10/748.abstract

HAMEL C. (2003). L'émergence d'une communauté professionnelle d'apprentissage et son accompagnement en réseau. Essai présenté à la Faculté des sciences de l'Université Laval pour l'obtention du grade de maître es arts.

HAMEL C. (2007). The third contribution in a thread : Nature and patterns. Toronto, ON: Knowledge Building Summer Institute (OISE).

HAMEL C. (2011). Prise de décisions individuelles et partagées des intervenants participant à l'École éloignée en réseau en matière d'innovation technologique, organisationnelle et sociale en région. Université Laval.

HAMEL C., ALLAIRE S., TURCOTTE S. (2012). Just-in-time online professional development activities for an innovation in small rural schools. Canadian Journal of Learning and Technology/La revue canadienne de l'apprentissage et de la technologie, Vol. $38 \mathrm{n}^{\circ} 3$.

HAMEL C., C.-BOUFFARD M.-H., ALLAIRE S., LAFERRIÈRE T., TURCOTTE S. (2006). Building capacity in the use of Knowledge Forum by identifying different types of discourses. Toronto : Knowledge Building Summer Institute (OISE).

HAMEL C., TURCOTTE S., LAFERRIÈRE T. (2012). Knowledge Forum uses for the improvement of explanation skills. Toronto, ON : Knowledge Building Summer Institute (OISE).

HAMEL C., TURCOTTE S., LAFERRIÈRE T. (2013). Evolution of the conditions for successful innovation in Remote Networked Schools. International Education Studies, Vol. 6 n³, p. 1-14. doi:10.5539/ies.v6n3p1

HAWLEY W., VALLI L. (2007). Design principles for learner-centered professional development. In W. H. with D. Rollie (Ed.), The keys to effective schools: Educational reform as continuous improvement (Thousands ., p. 117-137). Corwin, CA.

HMELO-SILVER C., BARROWS B. S. (2008). Facilitating collaborative knowledge building. Cognition and Instruction, Vol. 26, p. 48-94. doi:10.1080/07370000701798495

HUNZICKER J. (2012). Professional development in education professional development and job- embedded collaboration : How teachers learn to exercise leadership. Professional Development in Education, Vol. 38 n², p. 37-41.

INGVARSON L. (2005). Factors affecting the impact of professional development programs on teachers' knowledge, practice, student outcomes \& efficacy. Education Policy Analysis Archives, Vol. 13 nº10.

KRIPPENDORFF K. (2004). Content analysis: An introduction to its methodology (Sage.). Thousand Oaks.

LAFERRIÈRE T., ALLAIRE S., BREULEUX A., HAMEL C., TURCOTTE S., GAUDREAULT - PERRON J., ... INCHAUSPÉ P. (2008). L'école éloignée en réseau: L'apprentissage des élèves (p. 1-71). Québec. Disponible sur internet : http://www.cefrio.qc.ca/fileadmin/documents/Projets/L_Ecole_eloignee_en_reseau_ Phase3_Final_isbn.pdf

LAFERRIÈRE T., BARMA S., GERVAIS F., HAMEL C., ALLAIRE S., BREULEUX A. (2012). Teaching, learning and knowledge building: The case of the Remote Networked school initiative. Problems of Education in the 21st Century, Vol. 40, p. 96-113. Disponible sur internet http://journals.indexcopernicus.com/abstracted.php?level=5\&icid=988491 
LAFERRIÈRE T., BREULEUX A., HAMEL C., ALLAIRE S. (2004). L'École éloignée en réseau : Une contribution au maintien et au développement des petites écoles de village ( $p$. 43).

LAFERRIÈRE T., BREULEUX A., INCHAUSPÉ P. (2004). Rapport de recherche final du projet L'École éloignée en réseau (p. 168). CEFRIO et ministères de l'Éducation et des Régions

LAFERRIÈRE T., HAMEL C., ALLAIRE S., TURCOTTE S., BREULEUX A., BEAUDOIN J., GAUDREAULT-PERRON J. (2011). L'École éloignée en réseau (ÉÉR), un modèle (p. 37). Québec.

LAFERRIÈRE T., LAMON M. (2010). IRFI as a form of progressive discourse in knowledge building oriented classrooms. Summer Institute, 1-6. Disponible sur internet : http://ikit.org/SummerInstitute2010/doc/27-Laferriere-Lamon.pdf

LIEBERMAN A., POINTER MACE D. H. (2008). Teacher Learning: the Key to Educational Reform. Journal of Teacher Education, Vol. 59 n $^{\circ} 3$, p. 226-234. doi:10.1177/0022487108317020

MARX R. W., BLUMENFELD P., KRAJCIK J. S. (1998). New technologies for teacher professional development. Teaching and Teacher Education, Vol. 14, p. 33-52

MEQ. (2006). Le programme de formation de l'école québécoise (p. 362). Québec: Ministère de l'Éducation.

PENUEL W. R., FISHMAN B. J., YAMAGUCHI R., GALLAGHER L. P. (2007). What makes professional development effective? Strategies that foster curriculum implementation. American Educational Research Journal, Vol. 44 n $^{\circ} 4$, p. 921-958. doi:10.3102/0002831207308221

QUICK H. E., HOLTZMAN D. J., CHANEY K. R. (2009). Professional development and instructional practice: Conceptions and evidence of effectiveness. Journal of Education for Students Placed at Risk (JESPAR), Vol. $14 \mathrm{n}^{\circ} 1$, p. 45-71. doi:10.1080/10824660802715429

SCARDAMALIA M. (2002). Collective cognitive responsibility for the advancement of knowledge. In B. Smith (Ed.), Liberal education in a knowledge society, p. 67-98. Chicago, IL:Open Court.

SCARDAMALIA M., BEREITER C. (1991). Higher levels of agency for children in knowledge building: A challenge for the design of new knowledge media. Journal of the learning sciences, Vol. $1 \mathrm{n}^{\circ} 1$, p. 37-68. doi:10.1207/s15327809jls0101

TATE M. L. (2009). Workshops: Extend learning beyond your presentation with these brain- friendly strategies. Journal of Staff Development, Vol. $30 \mathrm{n}^{\circ} 1$, p. 44-46.

TURCOTTE S. (2008). Computer-supported collaborative inquiry in Remote Networked Schools. Argument. McGill University.

TURCOTTE S. (2012). Computer-supported collaborative inquiry on buoyancy: A discourse analysis supporting the "pieces" position on conceptual change. Journal of Science Education and Technology, Vol. 21 n6, p. 808-825. doi:10.1007/s10956012-9368-x

TURCOTTE S., HAMEL C. (2008). Necessary conditions to implement innovation in remote networked schools: The stakeholders' perceptions. Canadian Journal of Learning and Technology/La revue canadienne de l'apprentissage et de la technologie, Vol. $34 \quad \mathrm{n}^{\circ} 1$. Disponible sur internet : http://cjlt.csj.ualberta.ca/index.php/cjlt/article/view/176/172

TURCOTTE S., HAMEL C. (2011). Collaborer à des fins d'apprentissage en science et technologie au primaire : un accompagnement pédagogique en réseau significatif pour le développement professionnel des enseignants. Revue de l'éducation à distance, Vol. 25 n¹, p. 1-13. 
TURCOTTE S., HAMEL C., LAFERRIÈRE T. (2011). Investigating the use of the Knowledge Forum to improve student explanation skills: The case of the Remote Networked Schools. Hong Kong: Fourth World Universities Forum, The Hong Kong Institute of Education.

TURCOTTE S., LAFERRIÈRE T., HAMEL C., BREULEUX A. (2009). Multilevel innovation in Remote Networked Schools. Systemic Practice and Action Research, Vol. $23 \mathrm{n}^{\circ} 4$, p. 285-299. doi:10.1007/s11213-009-9160-x

UWAMARIYA A., MUKAMURERA J. (2005). Le concept de " développement professionnel » en enseignement : approches théoriques. Revue des sciences de l'éducation, Vol. $31 \mathrm{n}^{\circ} 1$, p. 133. doi:10.7202/012361ar

VILLEGAS-REIMERS E. (2003). Teacher professional development: an international review of the literature. Paris : Unesco. Disponible sur internet : http://www.cndwebzine.hcp.ma/cnd_sii/IMG/pdf/HTTP_ 4.PDF

WAYNE A. J., YOON K. S., ZHU P., CRONEN S., GARET M. S. (2008). Experimenting with teacher professional development: Motives and methods. Educational Researcher, Vol. 37 nº, p. 469-479. doi:10.3102/0013189X08327154

${ }^{1}$ Par dispositif, il est entendu l'ensemble des efforts qui sont mis en œuvre pour participer à l'apprentissage professionnel des enseignants.

${ }^{2}$ Il s'agit d'un organisme indépendant qui gère un ensemble d'écoles primaires et secondaires publiques sur un territoire géographique donné. Au Québec, il n’y a pas de découpage en fonction de la religion, mais il y a un découpage entre les commissions scolaires anglophones et francophones.

${ }^{3}$ Les conditions d'Ely $(1990,1999)$ sont : 1) L'insatisfaction face à la situation actuelle, 2) Connaissances et habiletés nécessaires, 3) Disponibilité de temps, 4) Disponibilité de ressources, 5) Incitatifs (octroi d'un portable), 6) Leadership, 7) Engagement des dirigeants, 8) Disponibilité de temps.

${ }^{4}$ À cet égard, nous n'avons pas documenté les activités de développement professionnel juste-au-cas bien que nous sachions qu'elles ont été existantes dans plusieurs commissions scolaires, ce sont les commissions scolaires qui ont elles-mêmes évalué ces activités. Nous n'excluons cependant pas qu'elles aient concouru au développement professionnel des enseignants participants.

${ }^{5}$ Il s'agit d'une version alternative du test international standardisé utilisé par le PISA.

${ }^{6} \mathrm{Au}$ départ, ces sessions de transfert servaient à développer le modèle de l'ÉÉR et à présenter des résultats de recherche de même que des pratiques exemplaires en développement. Au fil des ans, les enseignants sont devenus les principaux acteurs des sessions de transfert et ils présentent conjointement avec des collègues ou des chercheurs des activités réalisées, mais aussi des résultats de recherche en lien avec les dites activités.

7 Ce calcul considère la proportionnalité du nombre de participants dans les deux phases.

${ }^{8}$ Les itérations sont des activités de retour sur les résultats de recherche. Elles sont effectuées régulièrement et sont gérées de manière à éclairer la prise de décision des acteurs sur le terrain. Il en est question plus tard dans le texte.

${ }^{9}$ Les groupes contrôles étaient des classes qui possédaient pratiquement les mêmes caractéristiques (isolement, défavorisation, classes multiâges), mais qui ne participaient pas à l'ÉÉR dans une même commission scolaire. 